\title{
STRETCHING CONVEX DOMAINS TO CAPTURE MANY LATTICE POINTS
}

\author{
NICHOLAS F. MARSHALL
}

\begin{abstract}
We consider an optimal stretching problem for strictly convex domains in $\mathbb{R}^{d}$ that are symmetric with respect to each coordinate hyperplane, where stretching refers to transformation by a diagonal matrix of determinant 1 . Specifically, we prove that the stretched convex domain which captures the most positive lattice points in the large volume limit is balanced: the $(d-1)$-dimensional measures of the intersections of the domain with each coordinate hyperplane are equal. Our results extend those of Antunes \& Freitas, van den Berg, Bucur \& Gittins, Ariturk \& Laugesen, van den Berg \& Gittins, and Gittins \& Larson. The approach is motivated by the Fourier analysis techniques used to prove the classical $\#\left\{(i, j) \in \mathbb{Z}^{2}: i^{2}+j^{2} \leq r^{2}\right\}=$ $\pi r^{2}+\mathcal{O}\left(r^{2 / 3}\right)$ result for the Gauss circle problem.
\end{abstract}

\section{INTRODUCTION}

1.1. Introduction. In [1] Antunes \& Freitas introduced a new type of lattice point problem; namely, among all ellipses that are symmetric about the coordinate axes and of fixed area, which captures the most positive lattice points? More precisely, for $r \geq 1$ what is:

$$
a(r)=\underset{a>0}{\operatorname{argmax}} \#\left\{(i, j) \in \mathbb{Z}_{>0}^{2}:\left(\frac{i}{a}\right)^{2}+(j a)^{2} \leq r^{2}\right\} .
$$

Determining $a(r)$ for fixed $r \geq 1$ is challenging, even computationally [1]; however, Antunes \& Freitas were able to prove that

$$
\lim _{r \rightarrow \infty} a(r)=1,
$$

i.e., the ellipse which captures the most positive lattice points for large areas approaches a circle. Moreover, a rate of convergence of at least

$$
|a(r)-1|=\mathcal{O}\left(r^{-1 / 6}\right) \quad \text { as } r \rightarrow \infty,
$$

was established in [10] (and implied by Section 5 in [1]). This lattice point counting problem was

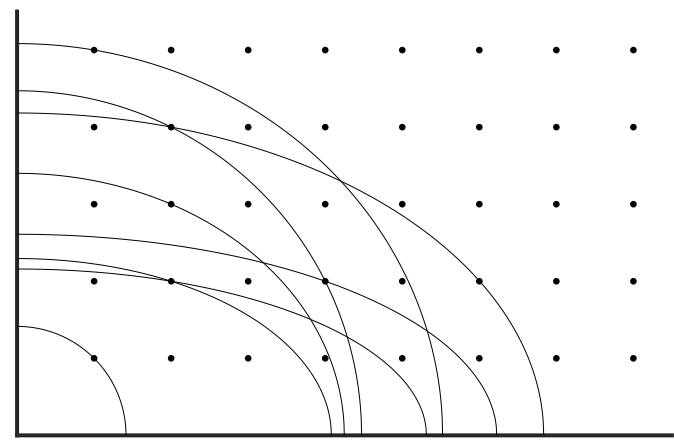

Figure 1. Eight ellipses which each capture the maximum possible number of positive lattice points for their given area.

originally motivated by a problem in high frequency shape optimization. Suppose that $\Omega \subset \mathbb{R}^{2}$ is

2010 Mathematics Subject Classification. 42B10, $52 \mathrm{C} 07$ (primary) and 11H06, 35P15, 90C27 (secondary).

Key words and phrases. Lattice point counting, shape optimization, decay of Fourier transform. 
an $a \times 1 / a$ rectangular domain. Then the eigenvalues of the Dirichlet Laplacian $-\Delta_{\Omega}^{\mathcal{D}}$ are of the form

$$
\sigma\left(-\Delta_{\Omega}^{\mathcal{D}}\right)=\left\{\pi^{2}\left(\left(\frac{i}{a}\right)^{2}+(a j)^{2}\right): i, j \in \mathbb{Z}_{>0}\right\}
$$

Thus, there is a bijection between the Dirichlet eigenvalues less than $\pi^{2} r^{2}$, and the positive lattice points in the ellipse $(x / a)^{2}+(a y)^{2} \leq r^{2}$. Hence, the statement $\lim _{r \rightarrow \infty} a(r)=1$ can also be interpreted as the statement that the rectangle that minimizes the Dirichlet eigenvalues in the high frequency limit approaches the square.

1.2. Neumann eigenvalues. A dual result for eigenvalues of the Neumann Laplacian $-\Delta_{\Omega}^{\mathcal{N}}$ was established by van den Berg, Bucur \& Gittins [17, where as before $\Omega \subset \mathbb{R}^{2}$ is an $a \times 1 / a$ rectangular domain. The eigenvalues of $-\Delta_{\Omega}^{\mathcal{N}}$ are of the form

$$
\sigma\left(-\Delta_{\Omega}^{\mathcal{N}}\right)=\left\{\pi^{2}\left(\left(\frac{i}{a}\right)^{2}+(a j)^{2}\right): i, j \in \mathbb{Z}_{\geq 0}\right\}
$$

and hence, the Neumann eigenvalues less than $\pi^{2} r^{2}$ are in bijection with the nonnegative lattice points in the ellipse $(x / a)^{2}+(a y)^{2} \leq r^{2}$. In terms of a lattice point problem, van den Berg, Bucur \& Gittins proved that if

$$
a(r)=\underset{a>0}{\operatorname{argmin}} \#\left\{(i, j) \in \mathbb{Z}_{\geq 0}^{2}:\left(\frac{i}{a}\right)^{2}+(j a)^{2} \leq r^{2}\right\},
$$

then $\lim _{r \rightarrow \infty} a(r)=1$. That is, the ellipse which captures the least nonnegative lattice points in the large area limit approaches the circle; equivalently, the rectangle which maximizes the Neumann eigenvalues in the high frequency limit approaches the square.

1.3. Higher dimensions. Subsequently, the result for Dirichlet eigenvalues was generalized to three dimensions by Gittins \& van den Berg [18, and recently to $d$-dimensions for both the Dirichlet and Neumann cases by Gittins \& Larson [5]. Specifically, Gittins \& Larson show that in $\mathbb{R}^{d}$ the cuboid of unit measure which minimizes the Dirichlet eigenvalues in the high frequency limit approaches the cube, and similarly, that the cuboid of unit measure which maximizes the Neumann Laplacian eigenvalues in the high frequency limit approaches the cube. Both the Dirichlet and Neumann cases have corresponding lattice point problems analogous to the 2-dimensional case. The Dirichlet case corresponds to the following lattice point problem. Suppose $A=\operatorname{diag}\left(a_{1}, a_{2}, \ldots, a_{d}\right)$ is a positive diagonal matrix of determinant 1 . For $r \geq 1$, let

$$
A(r)=\underset{A}{\operatorname{argmax}} \#\left\{\left(i_{1}, i_{2}, \ldots, i_{d}\right) \in \mathbb{Z}_{>0}^{d}:\left(\frac{i_{1}}{a_{1}}\right)^{2}+\left(\frac{i_{2}}{a_{2}}\right)^{2}+\cdots+\left(\frac{i_{d}}{a_{d}}\right)^{2} \leq r^{2}\right\} .
$$

Then the result of [5] implies that $\lim _{r \rightarrow \infty}\|A-\mathrm{Id}\|_{\infty}=0$. Moreover, Gittins \& Larson [5] show the following error estimate holds for dimensions $d \geq 2$ :

$$
\|A-\operatorname{Id}\|_{\infty}=\mathcal{O}\left(r^{-\frac{d-1}{2(d+1)}}\right) \quad \text { as } r \rightarrow \infty,
$$

and furthermore, they show a slightly improved error rate holds for $d>5$ by applying a result of Götze [6]. In this paper, we establish a similar rate of convergence for general convex domains. We note that the Neumann case in $d$-dimensions has an analogous dual formulation to the 2dimensional case, and similar error estimates were established in [5]. We remark that results concerning the optimization of eigenvalues of the Laplacian with perimeter constraints have also been considered [2, 4, 16. In particular, Bucur \& Freitas [4 proved that any sequence of minimizers of the Dirichlet eigenvalues with a perimeter constraint converges to the unit disk in the high frequency limit, and that among $k$-sided polygons, any sequence of minimizers converges to the regular $k$-sided polygon in the high frequency limit. 
1.4. Convex and concave curves. Laugesen \& Liu [10] and Ariturk \& Laugesen [3] generalized the two dimensional case of the above lattice point counting problems to certain classes of convex and concave curves. In particular, their results imply that among $p$-ellipses $|x / a|^{p}+|a y|^{p}=r^{p}$ for $p \in(0, \infty) \backslash\{1\}$ the $p$-ball captures the most positive lattice points. More generally, Laugesen \& Liu and Ariturk \& Laugesen show that the curves which capture the most positive integer lattice points in the large area limit are balanced: the distance from the origin to their points of intersection with the $x$-axis and $y$-axis are equal.

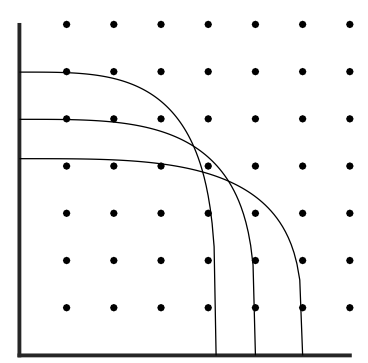

FIGURE 2. Among all stretches of a concave curve, which captures the most positive lattice points?

1.5. Right triangles. The results of [3, 10] exclude the $p=1$ case. In contrast to other values of $p$, for the $p=1$ case (where $p$-ellipses are right triangles) Ariturk \& Laugesen and Laugesen \& Liu conjectured that the optimal triangle

"does not approach a 45-45-90 degree triangle as $r \rightarrow \infty$. Instead one seems to get an infinite limit set of optimal triangles" (from [10]).

The author and Steinerberger 11 recently proved this conjecture and showed that the limit set is fractal of Minkowski dimension at most 3/4. Furthermore, all triangles which are optimal for infinitely many infinitely large areas have rational slopes, are contained in $[1 / 3,3]$, and have 1 as a unique accumulation point.
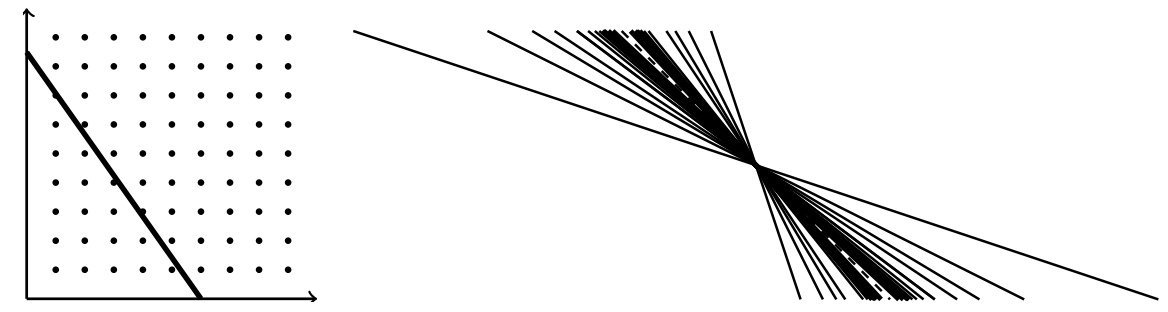

Figure 3. An illustration of the fractal set of infinitely many times optimal slopes.

1.6. Relation to spectral asymptotics. Suppose that $R \subset \mathbb{R}^{d}$ is a cuboid, and let $N_{R}(\lambda)$ denote the number of eigenvalues of the Dirichlet Laplacian $-\Delta_{R}^{\mathcal{D}}$ that are less than $\lambda$

$$
N_{R}(\lambda)=\#\left\{\mu \in \sigma\left(-\Delta_{R}^{\mathcal{D}}\right): \mu \leq \lambda\right\} .
$$

Then $N_{R}(\lambda)$ has a two-term asymptotic formula in terms of the volume $|R|$ and surface area $|\partial R|$ of $R$

$$
N_{R}(\lambda)=\frac{v_{d}}{(2 \pi)^{d}}|R| \lambda^{d / 2}-\frac{v_{d-1}}{4(2 \pi)^{d-1}}|\partial R| \lambda^{\frac{d-1}{2}}+o_{R}\left(\lambda^{\frac{d-1}{2}}\right), \quad \text { as } \quad \lambda \rightarrow \infty,
$$

where $v_{d}$ is the volume of the unit ball in $\mathbb{R}^{d}$. A similar asymptotic formula holds for the eigenvalues of the Neumann Laplacian $-\Delta_{R}^{\mathcal{N}}$, see [8]. We remark that in 1913, Weyl, see page 199 of [21, speculated about the existence of such a two-term asymptotic formula for more general domains in $\mathbb{R}^{3}$, and the problem of establishing the above two-term asymptotic formula for general domains became known as Weyl's Conjecture, see [8]. In 1980, Ivrii [9] proved that this two-term asymptotic 
formula indeed holds for more general domains under certain conditions. Suppose that $R$ is an $a_{1} \times a_{2} \times \cdots \times a_{d}$ cuboid. Then the eigenvalues of the Dirichlet Laplacian $-\Delta_{R}^{\mathcal{D}}$ are of the form

$$
\sigma\left(-\Delta_{R}^{\mathcal{D}}\right)=\left\{\pi^{2}\left(\left(\frac{i_{1}}{a_{1}}\right)^{2}+\left(\frac{i_{2}}{a_{2}}\right)^{2}+\cdots+\left(\frac{i_{d}}{a_{d}}\right)^{2}\right):\left(i_{1}, i_{2}, \ldots, i_{d}\right) \in \mathbb{Z}_{>0}^{d}\right\}
$$

and thus, the eigenvalues of $-\Delta_{R}^{\mathcal{D}}$ less than $\pi^{2} r^{2}$ are in bijection with the positive lattice points in the ellipsoid $\left(x_{1} / a_{1}\right)^{2}+\left(x_{2} / a_{2}\right)^{2}+\cdots+\left(x_{d} / a_{d}\right)^{2} \leq r^{2}$. If this cuboid $R$ has unit measure $|R|=1$, then its surface area $|\partial R|$ is given by

$$
|\partial R|=\sum_{j=1}^{d} 2 a_{j}^{-1}=2\left(\operatorname{tr} A^{-1}\right), \quad \text { where } \quad A=\operatorname{diag}\left(a_{1}, a_{2}, \ldots, a_{d}\right) .
$$

Substituting $\lambda=\pi^{2} r^{2}$ into the above two-term asymptotic formula for $N_{R}(\lambda)$ for this cuboid gives

$$
N_{R}\left(\pi^{2} r^{2}\right)=\frac{1}{2^{d}} v_{d} r^{d}-\frac{1}{2^{d}} v_{d-1}\left(\operatorname{tr} A^{-1}\right) r^{d-1}+o_{R}\left(r^{d-1}\right), \quad \text { as } \quad r \rightarrow \infty .
$$

We emphasize that the error term in this asymptotic formula depends implicitly on the cuboid $R$, and therefore, this asymptotic formula by itself is insufficient to determine which cuboid $R$ of unit measure maximizes $N_{R}(\lambda)$ as $\lambda \rightarrow \infty$. Addressing this issue is the main challenge in [1, 5, 17, 18, If we were to ignore the error term, then maximizing $N_{R}(\lambda)$ among cuboids of unit measure would be equivalent to minimizing $\operatorname{tr} A^{-1}$. By the arithmetic mean geometric mean inequality

$$
\operatorname{tr} A^{-1}=d \cdot \frac{a_{1}^{-1}+a_{2}^{-1}+\cdots+a_{d}^{-1}}{d} \geq d \cdot\left(a_{1}^{-1} \cdot a_{2}^{-1} \cdots \cdot a_{d}^{-1}\right)^{1 / d}=d,
$$

with equality if and only if $A=\mathrm{Id}$. Since $2 \operatorname{tr} A^{-1}=|\partial R|$, this inequality can be interpreted as an isoperimetric inequality for cuboids: the surface area of a cuboid of unit measure is greater than or equal to the surface area of the unit cube with equality if and only if the cuboid is the unit cube. Ultimately, after the main challenge of dealing with the error term has been appropriately handled, this isoperimetric inequality for cuboids is the reason that the cube is asymptotically optimal in [1, 15, 17, 18. In this paper, we consider a positive lattice point counting problem in more general domains; specifically, we study the number of positive lattice points in a fixed convex domain $\Omega$ that has been scaled by $r \geq 1$ and stretched by a linear transformation $A$ represented by a positive diagonal matrix of determinant 1 . In this generalized setting, the term $\operatorname{tr} A^{-1}$ similarly arises, and we use Fourier analysis to develop lattice point counting results which lead to uniform error estimates for optimal stretching problems.

1.7. Motivation. In this paper, our motivation is twofold: first, the results of Laugesen \& Liu and Ariturk \& Laugesen show that the asymptotic balancing observed in lattice point problems for ellipses [1, 5, 17, 18, extends from ellipses to a more general class of convex and concave curves, at least in two dimensions, and second, the analysis of the $p=1$ case in 11 shows that the convergence breaks down when the curves become flat. This phenomenon is common in harmonic analysis, where the decay of the Fourier transform is dependent on non-vanishing curvature. To briefly review the relation of Fourier analysis to lattice point problems, suppose $f$ is a $C^{\infty}$ function on $\mathbb{R}^{d}$ of compact support. Then the Poisson summation formula states that

$$
\sum_{n \in \mathbb{Z}^{d}} f(n)=\sum_{n \in \mathbb{Z}^{d}} \widehat{f}(n),
$$

where $\widehat{f}$ denotes the Fourier transform of $f$

$$
\widehat{f}(\xi)=\int_{\mathbb{R}^{d}} f(x) e^{-2 \pi i x \cdot \xi} d x .
$$

Suppose that $\chi(x)$ is the indicator function for a domain $\Omega \subset \mathbb{R}^{d}$. Then the indicator function for the scaled domain $r \Omega$ is $\chi_{r}(x)=\chi(x / r)$, whose Fourier transform, by a change of variables is

$$
\widehat{\chi}_{r}(\xi)=r^{d} \widehat{\chi}(r \xi) \text {. }
$$


Moreover, since $\widehat{\chi}_{r}(0)=|\Omega| r^{d}$, if the Poisson summation formula could be applied to $\chi_{r}$, it would express the number of lattice points inside $r \Omega$ as $|\Omega| r^{d}$ plus the sum of $\widehat{\chi}_{r}$ over the nonzero lattice points. Unfortunately, the Fourier transforms of indicator functions do not decay rapidly enough for the Poisson summation formula to be applied (these functions lack sufficient smoothness). However, this issue can be resolved by smoothing the indicator function by convolution with a bump function, and useful estimates can be obtained. This approach can be used to establish the classical

$$
\#\left\{(i, j) \in \mathbb{Z}^{2}: i^{2}+j^{2} \leq r^{2}\right\}=\pi r^{2}+\mathcal{O}\left(r^{2 / 3}\right),
$$

result for the Gauss circle problem accredited to Sierpiński [12, van der Corput [19, and Voronoi [20, which was the first non-trivial step towards the conjectured result:

$$
\#\left\{(i, j) \in \mathbb{Z}^{2}: i^{2}+j^{2} \leq r^{2}\right\}=\pi r^{2}+\mathcal{O}\left(r^{1 / 2+\varepsilon}\right),
$$

for all $\varepsilon>0$. Currently the best known result is $\mathcal{O}\left(r^{131 / 208}\right)$ due to Huxley [7. The argument for the $\mathcal{O}\left(r^{2 / 3}\right)$ error term for the circle in $\mathbb{R}^{2}$ can be generalized for convex domains $\Omega \subset \mathbb{R}^{d}$ whose boundary $\partial \Omega$ has nowhere vanishing Gauss curvature [13]. This generalization results in the following bound on the number of enclosed lattice points:

$$
\#\left\{n \in \mathbb{Z}^{d} \cap r \Omega\right\}=|\Omega| r^{d}+\mathcal{O}\left(r^{d-\frac{2 d}{d+1}}\right) .
$$

In this paper, we follow a similar approach to the proof of this result, but additionally handle the effects of stretching the domain. More specifically, given a strictly convex domain $\Omega$ which is symmetric with respect to each coordinate hyperplane, we consider the positive lattice points contained in the domain $A(r \Omega)=\{A(r x): x \in \Omega\}$, where $A$ is a positive diagonal matrix of determinant 1 , and $r \geq 1$ is a scaling factor.

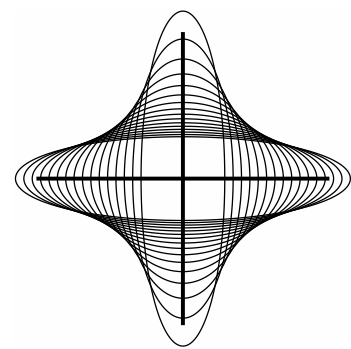

FIGURE 4 . The circle under the group action of positive diagonal matrices of determinant 1.

In the following we compute an asymptotic expansion for $\#\left\{n \in \mathbb{Z}^{d} \cap A(r \Omega)\right\}$ which includes both the effects of the diagonal transformation $A$ and scaling factor $r$; we use this expansion to derive uniform error estimates for an optimal stretching problem. The resulting theorem extends the results of [1, 3, 5, 17, 18. We note that we do not completely recover the results of [3, 10], since our approach can only represent curves which can be realized as the boundary of a convex domain whose boundary has nowhere vanishing Gauss curvature. However, there is some hope that the presented framework could be used to fully generalize the results of Laugesen \& Liu [10] and Ariturk \& Laugesen $[3$ by using more delicate bounds on the decay of the Fourier transform. Thus the presented approach may be useful for proving further generalizations.

\section{Main Result}

2.1. Main Result. Suppose that $\Omega \subset \mathbb{R}^{d}$ is a bounded convex domain whose boundary $\partial \Omega$ is $C^{d+2}$ and has nowhere vanishing Gauss curvature. Furthermore, suppose that $\Omega$ is symmetric with respect to each coordinate hyperplane and balanced in the following sense.

Definition 2.1. We say that a bounded convex domain $\Omega \subset \mathbb{R}^{d}$ is balanced if

$$
\left|\left\{\left(x_{1}, x_{2}, \ldots, x_{d}\right) \in \Omega: x_{j}=0\right\}\right|=1 \text { for } j=1,2, \ldots, d,
$$

where $|\cdot|$ denotes the $(d-1)$-dimensional Lebesgue measure. 
Note that the balanced assumption does not restrict the domains for which the below Theorem applies. Rather, the assumption that $\Omega$ is balanced is equivalent to choosing the unique balanced representative $B \Omega$ for $\Omega$, where $B$ is a positive diagonal matrix. Suppose that $A=\operatorname{diag}\left(a_{1}, a_{2}, \ldots, a_{d}\right)$ is a positive definite diagonal matrix of determinant 1 . To reiterate, we define

$$
A(r \Omega)=\{A(r x): x \in \Omega\},
$$

for scaling factor $r \geq 1$. That is to say, $A(r \Omega)$ is the domain $\Omega$ scaled by $r$ and transformed by $A$.
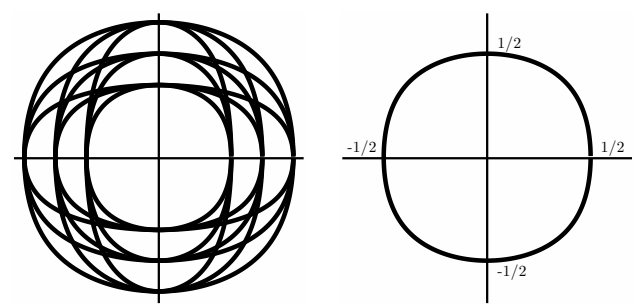

FigURE 5. Each domain $\Omega \subset \mathbb{R}^{d}$ which contains the origin has a unique balanced representative; the domains illustrated on the left share the balanced representative illustrated on the right.

Theorem 2.1. Suppose $\Omega \subset \mathbb{R}^{d}$ and $A: \mathbb{R}^{d} \rightarrow \mathbb{R}^{d}$ are as described above. Then for $d \geq 2$ and $r \geq 1$,

$$
\#\left\{n \in \mathbb{Z}_{>0}^{d} \cap A(r \Omega)\right\}=\frac{1}{2^{d}}|\Omega| r^{d}-\frac{1}{2^{d}}\left(\operatorname{tr} A^{-1}\right) r^{d-1}+\mathcal{O}\left(\left\|A^{-1}\right\|_{\infty}^{\frac{2 d}{d+1}} r^{d-\frac{2 d}{d+1}}\right),
$$

where the implicit constant is independent of $A$ and $r$. Moreover, if

$$
A(r)=\underset{A}{\operatorname{argmax}} \#\left\{n \in \mathbb{Z}_{>0}^{d} \cap A(r \Omega)\right\},
$$

then

$$
\|A(r)-\mathrm{Id}\|_{\infty}=\mathcal{O}\left(r^{-\frac{d-1}{2(d+1)}}\right) \quad \text { as } r \rightarrow \infty,
$$

where the implicit constant is independent of $A$ and $r$.

In the following remark, we describe specifically how the implicit constants in Theorem 2.1 depend on $\Omega \subset \mathbb{R}^{d}$, and provide conditions under which the expansion and convergence rate results in Theorem 2.1 hold uniformly over a family of domains.

Remark 2.1. The implicit constants in Theorem 2.1 which depend on the domain $\Omega \subset \mathbb{R}^{d}$ can be chosen in terms of the following three quantities: a lower bound on the Gauss curvature of $\Omega$, an upper bound on the diameter of $\Omega$, and a lower bound on the inradius of $\Omega$. Therefore, the result of Theorem 2.1 holds uniformly over any family of admissible domains, which have uniform bounds for these three quantities. Indeed, constants depending on $\Omega \subset \mathbb{R}^{d}$ enter the proof of Theorem 2.1 from three sources. First, we use a constant $C>0$ such that $\Omega \subset[-C, C]^{d}$; since $\Omega$ contains the origin it suffices to choose $C$ equal to the diameter of $\Omega$. Second, we use a constant $c>0$ from Lemma 3.1; by the proof of Lemma 3.1, this constant can be chosen as one divided by the inradius of $\Omega$. Third, we implicitly use a constant when using the bound in Lemma 3.3 for the decay of the Fourier transform of the indicator function for $\Omega$; this implicit constant can be chosen in terms of a lower bound on the Gauss curvature of the domain, cf. [13].

When $\Omega \subset \mathbb{R}^{d}$ is a $d$-dimensional ellipsoid, the Theorem implies the Dirichlet Laplacian results of Gittins \& Larson [5]. Applying the Theorem for dimension $d=2$ recovers the original result of Antunes \& Freitas [1] and agrees with the error estimate in [10. Specifically, in dimension $d=2$, the set of all positive diagonal matrices of determinant 1 is the 1 -parameter family $A=\operatorname{diag}(1 / a, a)$ for $a>0$ and the result of the Theorem can be stated as: 
Corollary 2.1. In the case $\Omega \subset \mathbb{R}^{2}$, the Theorem gives

$$
\#\left\{n \in \mathbb{Z}_{>0}^{2} \cap A(r \Omega)\right\}=\frac{1}{4}|\Omega| r^{2}-\frac{1}{4}\left(a+\frac{1}{a}\right) r+\mathcal{O}\left(a^{4 / 3} r^{2 / 3}\right),
$$

and

$$
|a(r)-1|=\mathcal{O}\left(r^{-1 / 6}\right) .
$$

As a second Corollary we can establish dual results for the nonnegative lattice point problem, which corresponds to the Neumann Laplacian results of Gittins \& Larson [5].

Corollary 2.2. Suppose $\Omega \subset \mathbb{R}^{d}$ and $A: \mathbb{R}^{d} \rightarrow \mathbb{R}^{d}$ satisfy the hypotheses of the Theorem, and additionally suppose $1 \leq\left\|A^{-1}\right\|_{\infty} \leq C r$ for a fixed constant $C>0$. Then

$$
\#\left\{n \in \mathbb{Z}_{\geq 0}^{d} \cap A(r \Omega)\right\}=\frac{1}{2^{d}}|\Omega| r^{d}+\frac{1}{2^{d}}\left(\operatorname{tr} A^{-1}\right) r^{d-1}+\mathcal{O}\left(\left\|A^{-1}\right\|_{\infty}^{\frac{2 d}{d+1}} r^{d-\frac{2 d}{d+1}}\right),
$$

where the implicit constant is independent of $A$ and $r$. Moreover, if

$$
A(r)=\underset{A}{\operatorname{argmin}} \#\left\{n \in \mathbb{Z}_{\geq 0}^{d} \cap A(r \Omega)\right\},
$$

then

$$
\|A(r)-\operatorname{Id}\|_{\infty}=\mathcal{O}\left(r^{-\frac{d-1}{2(d+1)}}\right)
$$

where the implicit constant is independent of $A$ and $r$.

The assumption $1 \leq\left\|A^{-1}\right\|_{\infty} \leq C r$ is needed for the expansion to hold, but not necessary for the convergence results as it serves to avoid the case where $A(r \Omega)$ contains more than order $r^{d}$ lattice points on the coordinate hyperplanes which is clearly non-optimal for the argmin. The proof of this Corollary follows from the arguments in Step 3.3 of the proof of Theorem 2.1

2.2. Generalization. In the following we describe a generalization of Theorem 2.1. In particular, we remark how Theorem 2.1 can be generalized to domains $\Omega$ which are not necessarily symmetric with respect to each coordinate hyperplane by considering the lattice points $\left\{n \in \mathbb{Z}^{d}: n_{j} \neq 0, \forall j=\right.$ $1, \ldots, d\}$ rather than the positive lattice points $\mathbb{Z}_{>0}^{d}$.

Remark 2.2. Suppose that $\Omega \subset \mathbb{R}^{d}$ is a bounded convex domain whose boundary $\partial \Omega$ is $C^{d+2}$ and has nowhere vanishing Gauss curvature. Moreover, suppose $\Omega$ is balanced in the sense of Definition 2.1, and contains the origin. Define $\mathbb{Z}_{\neq 0}^{d}:=\left\{n \in \mathbb{Z}^{d}: n_{j} \neq 0, \forall j=1, \ldots, d\right\}$. Let $A=\operatorname{diag}\left(a_{1}, a_{2}, \ldots, a_{d}\right)$ be a positive diagonal matrix of determinant 1 . Then

$$
\#\left\{n \in \mathbb{Z}_{\neq 0}^{d} \cap A(r \Omega)\right\}=|\Omega| r^{d}-\left(\operatorname{tr} A^{-1}\right) r^{d-1}+\mathcal{O}\left(\left\|A^{-1}\right\|_{\infty}^{\frac{2 d}{d+1}} r^{d-\frac{2 d}{d+1}}\right),
$$

where the implicit constant is independent of $A$ and $r$. Moreover, if

$$
A(r)=\underset{A}{\operatorname{argmax}} \#\left\{n \in \mathbb{Z}_{\neq 0}^{d} \cap A(r \Omega)\right\},
$$

then

$$
\|A(r)-\operatorname{Id}\|_{\infty}=\mathcal{O}\left(r^{-\frac{d-1}{2(d+1)}}\right)
$$

where the implicit constant is independent of $A$ and $r$. The proof of this statement is immediate from Step 3.3 of the proof of Theorem 2.1.

\section{Proof of main Result}

3.1. Proof strategy. Before discussing the technical details, we describe the proof strategy. The proof is divided into five steps. First, we establish a new lattice point counting result which holds uniformly over a family of positive diagonal matrices $A$ with determinant 1 . Specifically, we show that

$$
\#\left\{n \in \mathbb{Z}^{d} \cap A(r \Omega)\right\}=|\Omega| r^{d}+\mathcal{O}\left(\left\|A^{-1}\right\|_{\infty}^{\frac{2 d}{d+1}} r^{d-\frac{2 d}{d+1}}\right),
$$

when $1 \leq\left\|A^{-1}\right\|_{\infty} \leq C r$ where $C>0$ is a fixed constant. The key observation in the proof of this expansion is that the classical Fourier analysis techniques used to study the Gauss circle problem 
can be applied if the indicator function of the domain $A(r \Omega)$ is mollified by a bump function which has been appropriately stretched and scaled. Second, as a consequence of this result we show that the number of lattice points on the coordinate hyperplanes is, as expected, equal to $\left(\operatorname{tr} A^{-1}\right) r^{d-1}$ plus an appropriate error term; specifically,

$$
\#\left\{n \in \mathbb{Z}^{d} \cap A\left(r \bigcup_{j=1}^{d} \Omega_{j}\right)\right\}=\left(\operatorname{tr} A^{-1}\right) r^{d-1}+\mathcal{O}\left(\left\|A^{-1}\right\|_{\infty}^{\frac{2 d}{d+1}} r^{d-\frac{2 d}{d+1}}\right),
$$

where $\Omega_{j}$ denotes the intersection of $\Omega$ with the hyperplane orthogonal to the $j$-th coordinate vector. Third, we combine these two results to produce a positive lattice point counting result for $A(r \Omega)$ :

$$
\#\left\{n \in \mathbb{Z}_{>0}^{d} \cap A(r \Omega)\right\}=\frac{1}{2^{d}}|\Omega| r^{d}-\frac{1}{2^{d}}\left(\operatorname{tr} A^{-1}\right) r^{d-1}+\mathcal{O}\left(\left\|A^{-1}\right\|_{\infty}^{\frac{2 d}{d+1}} r^{d-\frac{2 d}{d+1}}\right) .
$$

Fourth, we show that this positive lattice point counting result implies that

$$
\|A(r)-\mathrm{Id}\| \rightarrow 0, \quad \text { as } r \rightarrow \infty, \quad \text { where } \quad A(r)=\underset{A}{\operatorname{argmax}} \#\left\{n \in \mathbb{Z}_{>0}^{d} \cap A(r \Omega)\right\},
$$

where the argmax is taken over positive diagonal matrices $A$ of determinant 1 . We note that clearly $\left\|A(r)^{-1}\right\|_{\infty}=\mathcal{O}(r)$ since otherwise $A(r \Omega)$ will not contain any positive lattice points; we proceed by considering two cases. First, we consider the case where

$$
\left\|A^{-1}\right\|_{\infty} \text { is on the order of } r
$$

where our positive lattice point counting result for $A(r \Omega)$ provides no information because the error term is order $r^{d}$. However, in such an extreme situation we are able to independently show non-optimality. Second, we assume that

$$
\left\|A^{-1}\right\|_{\infty}=o(r)
$$

and use the positive lattice point counting result to show convergence. Specifically, we assume

$$
\left\|A^{-1}\right\|_{\infty}=\psi(r) r, \quad \text { where } \quad \psi(r) \rightarrow 0, \quad \text { as } \quad r \rightarrow \infty .
$$

Substituting $\left\|A^{-1}\right\|_{\infty}=\psi(r) r$ into the positive lattice point counting result for $A(r \Omega)$ gives

$$
\#\left\{n \in \mathbb{Z}_{>0}^{d} \cap A(r \Omega)\right\} \leq \frac{1}{2^{d}}|\Omega| r^{d}-\left(\frac{1}{2^{d}}-C \psi(r)^{\frac{d-1}{d+1}}\right) \psi(r) r^{d},
$$

where $C$ is a fixed constant. Since $\psi \rightarrow 0$ the term in the parentheses we eventually be positive, and hence, comparison to the case where $A=\mathrm{Id}$ implies convergence. Fifth, and finally, given the fact that $A(r)$ converges to Id we establish the convergence rate

$$
\|A(r)-\operatorname{Id}\|_{\infty}=\mathcal{O}\left(r^{-\frac{d-1}{2(d+1)}}\right)
$$

using a standard arithmetic mean geometric mean argument.

3.2. Useful lemmata. The proof of the main result relies on three lemmata: two geometric in nature, and one related to the decay of the Fourier transform of indicator functions of convex domains with nowhere vanishing Gauss curvature. Lemmata 3.1 and 3.2 are proved in this section, and are motivated by similar technical results in [14, while Lemma 3.3 appears in standard references, cf., [13, 14, 15].

Lemma 3.1. Suppose that $\Omega \subset \mathbb{R}^{d}$ is a bounded convex open domain which contains the origin. Then there exists a constant $c>0$ such that for all $0<r<\infty$, all $0<\delta<\infty$, and all $0 \leq|y| \leq \delta$

$$
x \in r \Omega \Longrightarrow x+y \in(r+c \delta) \Omega \text {. }
$$

Proof. Since $\Omega$ is open, bounded, and contains the origin $\overrightarrow{0} \in \mathbb{R}^{d}$, there exists a constant $\varepsilon>0$ such that

$$
\left\{x \in \mathbb{R}^{d}:|x-\overrightarrow{0}| \leq \varepsilon\right\} \subset \Omega .
$$


Set $c=1 / \varepsilon$, and suppose that $0<r<\infty, x \in r \Omega$, and $0<|y| \leq \delta$ are given. Then

$$
y=\frac{|y|}{\varepsilon} \cdot \varepsilon \frac{y}{|y|} \in\left\{x \in \mathbb{R}^{d}:|x-\overrightarrow{0}| \leq c \delta \varepsilon\right\} \subseteq c \delta \Omega
$$

and therefore,

$$
x+y \in r \Omega+c \delta \Omega \subseteq(r+c \delta) \Omega,
$$

where the final inclusion follows from the convexity of $\Omega$.

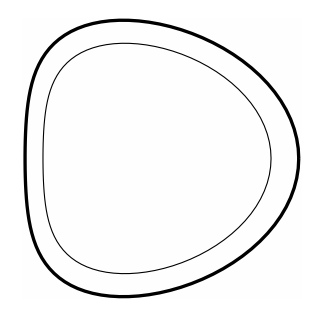

Figure 6. Geometrically Lemma 3.1 implies that $\left\{x \in \mathbb{R}^{d}: d(x, r \Omega) \leq \delta\right\} \subseteq(r+c \delta) \Omega$.

The application of this Lemma to lattice point counting problems occurs when developing bounds for indicator functions in terms of mollified indicator functions. Specifically, Lemma 3.1 is typically applied as follows. Suppose $\chi$ is the indicator function for the set $\Omega \subset \mathbb{R}^{d}$, and define

$$
\chi_{r}(x)=\chi(x / r),
$$

which is the indicator function for $r \Omega$. Let $\varphi$ denote a $C^{\infty}$ bump function supported on the unit ball which integrates to 1 . Set

$$
\varphi_{\delta}(x)=\delta^{-d} \varphi(x / \delta) .
$$

Suppose $c>0$ is chosen in accordance to Lemma 3.1 . Then we claim that for all $x \in \mathbb{R}^{d}$

$$
\chi_{r}(x) \leq\left[\chi_{r+c \delta} * \varphi_{\delta}\right](x),
$$

where $*$ denotes convolution. Indeed, by the choice of $c>0$, for all $|y| \leq \delta$

$$
\chi_{r}(x)=1 \Longrightarrow \chi_{r+c \delta}(x-y)=1 \text {. }
$$

Therefore, if $\chi_{r}(x)=1$, then

$$
\left[\chi_{r+c \delta} * \varphi_{\delta}\right](x)=\int_{|y| \leq \delta} \chi_{r+c \delta}(x-y) \varphi_{\delta}(y) d y=\int_{|y| \leq \delta} \varphi_{\delta}(y) d y=1 .
$$

In the proof of the Theorem in the following section a similar result is required. However, in this case the indicator functions and bump functions under consideration are transformed by a positive diagonal linear transformation $A$ of determinant 1 . More precisely, we consider

$$
\chi_{A, r}(x)=\chi\left(A^{-1} x / r\right) \text { and } \varphi_{A, \delta}=\delta^{-d} \varphi\left(A^{-1} x / \delta\right),
$$

where $A$ is a positive diagonal matrix of determinant 1 . Let

$$
\chi_{A, r, \delta}=\chi_{A, r} * \varphi_{A, \delta} .
$$

In the following Lemma, we repeat the above analysis to develop upper and lower bounds for $\chi_{A, r}$ in terms of the smoothed version $\chi_{A, r, \delta}$.

Lemma 3.2. Suppose $\chi_{A, r}$ and $\chi_{A, r, \delta}$ are as defined above. Then there exists a constant $c>0$ such that for all $r>0$, all $\delta \leq r /(1+c)$, and all positive diagonal matrices $A$ of determinant 1

$$
\chi_{A, r-c \delta, \delta}(x) \leq \chi_{A, r}(x) \leq \chi_{A, r+c \delta, \delta}(x),
$$

for all $x \in \mathbb{R}^{d}$. 
Proof. By Lemma 3.1 we may choose a constant $c>0$ such that for all $r>0$ and all $\delta>0$

$$
x \in r \Omega \wedge|y| \leq \delta \Longrightarrow x+y \in(r+c \delta) \Omega .
$$

To establish the upper bound it suffices to show that $\chi_{A, r}(x)=1 \Longrightarrow \chi_{A, r+c \delta, \delta}=1$. By definition

$$
\chi_{A, r+c \delta, \delta}(x)=\int_{\left|A^{-1} y\right| \leq \delta} \chi_{r+c \delta}\left(A^{-1}(x-y)\right) \varphi_{\delta}\left(A^{-1} y\right) d y .
$$

Since $A$ is a diagonal matrix of determinant 1, by a change of variables of integration we conclude

$$
\int_{\left|A^{-1} y\right| \leq \delta} \chi_{r+c \delta}\left(A^{-1}(x-y)\right) \varphi_{\delta}\left(A^{-1} y\right) d y=\int_{|y| \leq \delta} \chi_{r+c \delta}\left(A^{-1} x-y\right) \varphi_{\delta}(y) d y .
$$

If $\chi_{A, r}(x)=1$, then by the choice of $c>0$ we have $\chi_{r+c \delta}\left(A^{-1} x-y\right)=1$, when $|y| \leq \delta$. Thus

$$
\int_{|y| \leq \delta} \chi_{r+c \delta}\left(A^{-1} x-y\right) \varphi_{\delta}(y) d y=\int_{|y| \leq \delta} \varphi_{\delta}(y) d y=1,
$$

which establishes the upper bound. Next, to establish the lower bound we show that $\chi_{A, r}(x)=$ $0 \Longrightarrow \chi_{A, r-c \delta, \delta}(x)=0$. Let $\tilde{x}=x-y$ and $\tilde{r}=r-c \delta$. By Lemma 3.1

$$
\tilde{x} \in \tilde{r} \Omega \wedge|y| \leq \delta \Longrightarrow \tilde{x}+y \in(\tilde{r}+c \delta) \Omega .
$$

Writing the contrapositive of this statement in terms of $x$ and $r$ gives

$$
x \notin r \Omega \wedge|y| \leq \delta \Longrightarrow x-y \notin(r-c \delta) \Omega .
$$

Using the same change of variables of integration as above, we have

$$
\chi_{A, r-c \delta, \delta}(x)=\int_{|y| \leq \delta} \chi_{r-c \delta}\left(A^{-1} x-y\right) \varphi_{\delta}(y) d y .
$$

If $\chi_{A, r}(x)=0$, then the derived implication implies that $\chi_{r-c \delta}\left(A^{-1} x-y\right)=0$ for all $|y| \leq \delta$; thus

$$
\int_{|y| \leq \delta} \chi_{r-c \delta}\left(A^{-1} x-y\right) \varphi_{\delta}(y) d y=0,
$$

which completes the proof.

Lemma 3.3. Suppose $\Omega \subset \mathbb{R}^{d}$ is a bounded convex domain with $C^{d+2}$ boundary $\partial \Omega$ with nowhere vanishing Gauss curvature. If $\chi$ is the indicator function of $\Omega$, then

$$
|\widehat{\chi}(\xi)|=\mathcal{O}\left(|\xi|^{-\frac{d+1}{2}}\right), \quad \text { as }|\xi| \rightarrow \infty .
$$

That is to say, the Fourier transform of an indicator function decays one order better than the Fourier transform of the corresponding surface carried measure $d \mu$, which decays like

$$
|\widehat{d \mu}|=\mathcal{O}\left(|\xi|^{-\frac{d-1}{2}}\right) .
$$

The proof of this lemma involves an integration by parts of an expression for the Fourier transform of the corresponding surface carried measure, which is where the extra order of convergence arises.

3.3. Proof of Theorem 2.1. For clarity, we have divided the proof of the Theorem into five steps. First, we fix notation. We say

$$
f(x) \lesssim_{h} g(x) \text { if and only if } f(x) \leq C_{h} g(x),
$$

for a fixed constant $C_{h}>0$ only depending on $h$. Throughout the proof, we assume that $r \geq 1$, $d \geq 2$, and that $\Omega \subset \mathbb{R}^{d}$ is a bounded convex domain with a $C^{d+2}$ boundary $\partial \Omega$ with nowhere vanishing Gauss curvature. In particular, we assume that

$$
\Omega \subset[-C, C]^{d} \text { where } C>0 \text { is a fixed constant. }
$$

Let $A=\operatorname{diag}\left(a_{1}, a_{2}, \ldots, a_{d}\right)$ denote a positive diagonal matrix of determinant 1 . Without loss of generality we may assume that $a_{1} \leq a_{2} \leq \ldots \leq a_{d}$, and set $a=1 / a_{1}=\left\|A^{-1}\right\|_{\infty}$. 
Step 3.1. Suppose $1 \leq a \leq C r$. Then

$$
\#\left\{n \in \mathbb{Z}^{d} \cap A(r \Omega)\right\}=|\Omega| r^{d}+\mathcal{O}\left(a^{\frac{2 d}{d+1}} r^{d-\frac{2 d}{d+1}}\right),
$$

where the implicit constant is independent of $A$ and $r$.

Proof. Let $\chi$ denote the indicator function for the given domain $\Omega$, and set

$$
\chi_{A, r, \delta}(x)=\left[\chi_{A, r} * \varphi_{A, \delta}\right](x),
$$

where $\chi_{A, r}=\chi\left(A^{-1} x / r\right)$ and $\varphi_{A, \delta}=\delta^{-d} \varphi\left(A^{-1} x / \delta\right)$ for some $C^{\infty}$ bump function $\varphi$ supported on the unit ball in $\mathbb{R}^{d}$ and such that

$$
\int_{\mathbb{R}^{d}} \varphi(x) d x=1 .
$$

We denote our "smoothed" approximation of the number of lattice points enclosed by $A(r \Omega)$ by

$$
N_{A, r, \delta}=\sum_{n \in \mathbb{Z}^{d}} \chi_{A, r, \delta}(n) .
$$

Since $\chi_{A, r, \delta}$ is $C^{\infty}$ and of compact support, by the Poisson summation formula

$$
N_{A, r, \delta}=\sum_{n \in \mathbb{Z}^{d}} \widehat{\chi}_{A, r, \delta}(n)=\sum_{n \in \mathbb{Z}^{d}} r^{d} \widehat{\chi}(A n r) \widehat{\varphi}(A n \delta) .
$$

Since $\widehat{\chi}(0)=|\Omega|$ and $\widehat{\varphi}(0)=1$, we can break the sum up into three parts:

$$
N_{A, r, \delta}=|\Omega| r^{d}+\sum_{0<|n|<a / \delta} r^{d} \widehat{\chi}(A n r) \widehat{\varphi}(A n \delta)+\sum_{|n| \geq a / \delta} r^{d} \widehat{\chi}(A n r) \widehat{\varphi}(A n \delta) .
$$

Since $\widehat{\chi}$ is the Fourier transform of an indicator function of a convex domain with nowhere vanishing Gauss curvature, it follows from Lemma 3.3 that

$$
|\widehat{\chi}(\xi)| \lesssim \Omega|\xi|^{-\frac{d+1}{2}} .
$$

Furthermore, since $\widehat{\varphi}$ is the Fourier transform of a $C^{\infty}$ function with compact support if follows that

$$
|\widehat{\varphi}(\xi)| \lesssim_{\varphi}|\xi|^{-N}
$$

for all $N \geq 0$. To evaluate the first sum we use the fact that $|\widehat{\chi}| \lesssim_{\Omega}|\xi|^{-\frac{d+1}{2}}$, and $|\widehat{\varphi}| \lesssim_{\varphi} 1$

$$
\sum_{0<|n| \leq a / \delta} r^{d} \widehat{\chi}(A n r) \widehat{\varphi}(A n \delta) \lesssim_{\Omega, \varphi} \sum_{0<|n| \leq a / \delta} r^{d} r^{-\frac{d+1}{2}} a^{\frac{d+1}{2}}|n|^{-\frac{d+1}{2}} .
$$

Therefore,

$$
\sum_{0<|n| \leq a / \delta} r^{d} \widehat{\chi}(A n r) \widehat{\varphi}(A n \delta) \lesssim \Omega, \varphi r^{\frac{d-1}{2}} a^{\frac{d+1}{2}} \sum_{0<|n| \leq a / \delta}|n|^{-\frac{d+1}{2}} .
$$

The sum on the right hand side can be compared to the integral

Thus we conclude that

$$
\int_{|x| \leq a / \delta}|x|^{-\frac{d+1}{2}} d x=C_{d} \int_{0}^{a / \delta} t^{-\frac{d+1}{2}} t^{d-1} d t=C_{d} a^{\frac{d-1}{2}} \delta^{-\frac{d-1}{2}} .
$$

$$
\sum_{0<|n| \leq a / \delta} r^{d} \widehat{\chi}(A n r) \widehat{\varphi}(A n \delta) \lesssim \Omega, \varphi r^{\frac{d-1}{2}} a^{d} \delta^{-\frac{d-1}{2}} .
$$

For the second sum, we use the fact that

$$
|\widehat{\varphi}(\xi)| \lesssim_{\varphi}|\xi|^{-\frac{d}{2}}
$$

and use the same decay of $\widehat{\chi}$ as before. This yields

$$
\sum_{|n| \geq a / \delta} r^{d} \widehat{\chi}(A n r) \widehat{\varphi}(A n \delta) \lesssim_{\Omega, \varphi} \sum_{|n| \geq a / \delta} r^{d} r^{-\frac{d+1}{2}} a^{\frac{d+1}{2}}|n|^{-\frac{d+1}{2}} a^{\frac{d}{2}}|n|^{-\frac{d}{2}} \delta^{-\frac{d}{2}} .
$$

Hewe

$$
\sum_{|n| \geq a / \delta} r^{d} \widehat{\chi}(A n r) \widehat{\varphi}(A n \delta) \lesssim \Omega, \varphi r^{\frac{d-1}{2}} \delta^{-\frac{d}{2}} a^{d+\frac{1}{2}} \sum_{|n| \geq a / \delta}|n|^{-d-\frac{1}{2}} .
$$


We can bound the sum on the right hand side by comparison to the integral

$$
\int_{|x| \geq a / \delta}|x|^{-d-1 / 2} d x=C_{d} \int_{a / \delta}^{\infty} t^{-d-\frac{1}{2}} t^{d-1} d t=C_{d} a^{-\frac{1}{2}} \delta^{\frac{1}{2}} .
$$

So

$$
\sum_{|n| \geq a / \delta} r^{d} \widehat{\chi}(A n r) \widehat{\varphi}(A n \delta) \lesssim \Omega, \varphi r^{\frac{d-1}{2}} a^{d} \delta^{-\frac{d-1}{2}} .
$$

Moreover, since the bound for both sums is the same

$$
\left|N_{A, r, \delta}-\right| \Omega\left|r^{d}\right| \lesssim_{\Omega, \varphi} r^{\frac{d-1}{2}} a^{d} \delta^{-\frac{d-1}{2}} .
$$

Set

$$
\delta=a^{\frac{2 d}{d+1}} r^{-\frac{d-1}{d+1}} .
$$

Substituting this value of $\delta$ into the last inequality yields

$$
\left|N_{A, r, \delta}-\right| \Omega\left|r^{d}\right| \lesssim_{\Omega, \varphi} a^{\frac{2 d}{d+1}} r^{d-\frac{2 d}{d+1}} .
$$

By Lemma 3.2 there exists a constant $c>0$ such that for all $r>0$, all $\delta \leq r /(1+c)$, and all positive diagonal matrices $A$ of determinant 1

$$
\chi_{A, r-c \delta, \delta}(x) \leq \chi_{A, r}(x) \leq \chi_{A, r+c \delta}(x),
$$

for all $x \in \mathbb{R}^{d}$. However, we do not in general know that $\delta \leq r /(1+c)$. Therefore, in the following we consider two cases:

$$
1 \leq a \leq(1+c)^{-\frac{d+1}{2 d}} r, \quad \text { and } \quad(1+c)^{-\frac{d+1}{2 d}} r<a \leq C r .
$$

Case 1. If $1 \leq a \leq(1+c)^{-\frac{d+1}{2 d}} r$, then

$$
\delta=a^{\frac{2 d}{d+1}} r^{-\frac{d-1}{d+1}} \Longrightarrow \delta \leq r /(1+c) .
$$

Thus, we may apply Lemma 3.2 to conclude

$$
\chi_{A, r-c \delta, \delta}(x) \leq \chi_{A, r}(x) \leq \chi_{A, r+c \delta, \delta}(x) .
$$

Therefore, by the definition of $N_{A, r, \delta}$

$$
N_{A, r-c \delta, \delta} \leq \#\left\{n \in \mathbb{Z}^{d} \cap A(r \Omega)\right\} \leq N_{A, r+c \delta, \delta} .
$$

Moreover, since $\delta \leq r /(1+c)$ applying the bound derived above gives

$$
\left|N_{A, r+c \delta, \delta}-\right| \Omega\left|r^{d}\right| \lesssim \Omega, \varphi a^{\frac{2 d}{d+1}} r^{d-\frac{2 d}{d+1}},
$$

and similarly,

$$
\left|N_{A, r-c \delta, \delta}-\right| \Omega\left|r^{d}\right| \lesssim \Omega, \varphi a^{\frac{2 d}{d+1}} r^{d-\frac{2 d}{d+1}} .
$$

Therefore, we conclude

$$
\left|\#\left\{n \in \mathbb{Z}^{d} \cap A(r \Omega)\right\}-\right| \Omega\left|r^{d}\right| \lesssim_{\Omega, \varphi} a^{\frac{2 d}{d+1}} r^{d-\frac{2 d}{d+1}} .
$$

Note $\varphi$ can be fixed such that it only depends on $\Omega$ (more specifically, dependent only on the dimension of $\Omega$ ), so the proof is complete for this case. 
Case 2. If $(1+c)^{-\frac{d+1}{2 d}} r<a \leq C r$, then we define

$$
\tilde{c}:=a(1+c)^{\frac{d+1}{2 d}} r^{-1} .
$$

Since the determinant of $A$ is equal to 1 , there exists $1<k<d$ such that

$$
a_{1} \leq \ldots \leq a_{k} \leq 1 \leq a_{k+1} \leq \ldots \leq a_{d} .
$$

Define

$$
\tilde{a}_{j}=\left\{\begin{array}{cc}
a_{j} \cdot \tilde{c}^{-1} & \text { for } 1 \leq j \leq k \\
a_{j} \cdot \tilde{c}^{\frac{k}{d-k}} & \text { for } k<j \leq d
\end{array} .\right.
$$

Suppose $\tilde{A}=\operatorname{diag}\left(\tilde{a}_{1}, \tilde{a}_{2}, \ldots, \tilde{a}_{d}\right)$. Then by construction

$$
\operatorname{det}(\tilde{A})=1 \quad \text { and } \quad\left\|\tilde{A}^{-1}\right\|_{\infty}=(1+c)^{-\frac{d+1}{2 d}} r .
$$

By the domain monotonicity of lattice point counting and the result from Case 1

$$
\#\left\{n \in \mathbb{Z}^{d} \cap A(r \Omega)\right\} \leq \#\left\{n \in \mathbb{Z}^{d} \cap \tilde{A}(\tilde{c} r \Omega)\right\}=\mathcal{O}\left((\tilde{c} r)^{d}\right) .
$$

Since $1 \leq \tilde{c} \leq c^{\frac{d+1}{2 d}} C$ and the constants $c$ and $C$ only depend on $\Omega$ we conclude that

$$
\#\left\{n \in \mathbb{Z}^{d} \cap A(r \Omega)\right\}=\mathcal{O}\left(r^{d}\right),
$$

where the implicit constant only depends on $\Omega$. This completes the proof of Step 3.1 .

Step 3.2. Suppose that $1 \leq a \leq C r$, and assume that $\Omega$ is balanced in the sense of Definition 2.1. Let $\Omega_{j}$ denote the intersection of $\Omega$ with the coordinate hyperplane orthogonal to the $j$-th coordinate vector. Then

$$
\#\left\{n \in \mathbb{Z}^{d} \cap A\left(r \bigcup_{j=1}^{d} \Omega_{j}\right)\right\}=\left(\operatorname{tr} A^{-1}\right) r^{d-1}+\mathcal{O}\left(a^{\frac{2 d}{d+1}} r^{d-\frac{2 d}{d+1}}\right),
$$

where the implicit constant is independent of $A$ and $r$.

Proof. If $d=1$, then the statement is trivial. We consider two cases: $d=2$ and $d>2$.

Case 1. If the dimension $d=2$, then the set of positive diagonal matrices of determinant 1 is the 1-parameter family $A=\operatorname{diag}(1 / a, a)$. Therefore, it suffices to show that

$$
\#\left\{n \in \mathbb{Z}^{2} \cap A\left(r \bigcup_{j=1}^{2} \Omega_{j}\right)\right\}=\left(a+\frac{1}{a}\right) r+\mathcal{O}\left(a^{\frac{4}{3}} r^{\frac{2}{3}}\right),
$$

for $A=\operatorname{diag}(1 / a, a)$. In this case, $\Omega_{1}$ and $\Omega_{2}$ are the intersection of $\Omega$ with the $y$-axis and $x$-axis, respectively. Moreover, since we have assumed $\Omega$ is balanced $\left|\Omega_{j}\right|=1$ for $j=1,2$. Therefore, when $\Omega$ is scaled by $r$ and transformed by $A$, the total number of points on the axes will be $a r+r / a+\mathcal{O}(1)$, and thus the above statement holds.

Case 2. Suppose the dimension $d>2$. Let $A_{j}$ denote the $(d-1) \times(d-1)$ positive diagonal matrix formed by removing the $j$-th row and $j$-th column from $A$. Write:

$$
\#\left\{n \in \mathbb{Z}^{d} \cap A_{j}\left(r \Omega_{j}\right)\right\}=\#\left\{n \in \mathbb{Z}^{d} \cap a_{j}^{\frac{1}{d-1}} A_{j}\left(r a_{j}^{-\frac{1}{d-1}} \Omega_{j}\right)\right\} .
$$

Observe that

$$
\operatorname{det} a_{j}^{\frac{1}{d-1}} A_{j}=1 \text { and }\left\|\left(a_{j}^{\frac{1}{d-1}} A_{j}\right)^{-1}\right\|_{\infty} \leq\left(a_{j}^{-\frac{1}{d-1}}\right) a .
$$

Therefore, by the result from Step 3.1

$$
\left|\#\left\{n \in \mathbb{Z}^{d} \cap a_{j}^{\frac{1}{d-1}} A_{j}\left(r a_{j}^{-\frac{1}{d-1}} \Omega_{j}\right)\right\}-\right| \Omega_{j}\left|a_{j}^{-1} r^{d-1}\right| \lesssim \Omega\left(a_{j}^{-\frac{1}{d-1}} a\right)^{\frac{2(d-1)}{d}}\left(a_{j}^{-\frac{1}{d-1}} r\right)^{(d-1)-\frac{2(d-1)}{d}} .
$$


Observe that the total contribution of $a_{j}$ to the right hand side of this inequality is

$$
a_{j}^{\left(-\frac{1}{d-1}\right)\left(\frac{2(d-1)}{d}+(d-1)-\frac{2(d-1)}{d}\right)}=a_{j}^{-1} .
$$

Therefore, bounding $a_{j}^{-1}$ by $a=\left\|A^{-1}\right\|_{\infty}$ gives

$$
\left|\#\left\{n \in \mathbb{Z}^{d} \cap a_{j}^{\frac{1}{d-1}} A_{j}\left(r a_{j}^{-\frac{1}{d-1}} \Omega_{j}\right)\right\}-\right| \Omega_{j}\left|a_{j}^{-1} r^{d-1}\right| \lesssim \Omega a \cdot\left(a^{\frac{2(d-1)}{d}} r^{(d-1)-\frac{2(d-1)}{d}}\right) .
$$

A direct computation shows that

$$
\left(a^{\frac{2 d}{d+1}} r^{d-\frac{2 d}{d+1}}\right)\left(a^{\frac{2(d-1)}{d}} r^{(d-1)-\frac{2(d-1)}{d}}\right)^{-1}=a^{\frac{2}{d^{2}+d}} r^{\frac{d^{2}+d-2}{d^{2}+d}} .
$$

Thus, since we have assumed that $a \leq C r$, it follows that

$$
a \cdot\left(a^{\frac{2(d-1)}{d}} r^{(d-1)-\frac{2(d-1)}{d}}\right) \lesssim \Omega a^{\frac{2 d}{d+1}} r^{d-\frac{2 d}{d+1}} .
$$

Therefore, we obtain the bound

$$
\left|\#\left\{n \in \mathbb{Z}^{d} \cap A_{j}\left(r \Omega_{j}\right)\right\}-\right| \Omega_{j}\left|a_{j}^{-1} r^{d-1}\right| \lesssim \Omega^{a^{\frac{2 d}{d+1}}} r^{d-\frac{2 d}{d+1}} .
$$

By the balanced assumption $\left|\Omega_{j}\right|=1$. Therefore, summing over $j=1, \ldots, d$ yields

$$
\left|\sum_{j=1}^{d} \#\left\{n \in \mathbb{Z}^{d} \cap A_{j}\left(r \Omega_{j}\right)\right\}-\left(\operatorname{tr} A^{-1}\right) r^{d-1}\right| \lesssim \Omega a^{\frac{2 d}{d+1}} r^{d-\frac{2 d}{d+1}}
$$

Next, we will use a similar argument to analyze $\mathbb{Z}^{d} \cap A_{j, k}\left(r \Omega_{j, k}\right)$, where $\Omega_{j, k}=\Omega_{j} \cap \Omega_{k}$ and $A_{j, k}$ denotes the $(d-2) \times(d-2)$ matrix formed by removing the $j$-th and $k$-th rows, and $j$-th and $k$-th columns from $A$. Write:

$$
\#\left\{n \in \mathbb{Z}^{d} \cap A_{j, k}\left(r \Omega_{j, k}\right)\right\}=\#\left\{n \in \mathbb{Z}^{d} \cap\left(a_{j} a_{k}\right)^{\frac{1}{d-2}} A_{j, k}\left(r\left(a_{j} a_{k}\right)^{-\frac{1}{d-2}} \Omega_{j}\right)\right\} .
$$

In this case, applying Step 3.1 yields:

$$
\begin{aligned}
\mid \#\{n \in & \left.\mathbb{Z}^{d} \cap\left(a_{j} a_{k}\right)^{\frac{1}{d-2}} A_{j, k}\left(r\left(a_{j} a_{k}\right)^{-\frac{1}{d-2}} \Omega_{j}\right)\right\}-\left|\Omega_{j, k}\right| a_{j}^{-1} a_{k}^{-1} r^{d-2} \mid \\
& \lesssim \Omega\left(\left(a_{j} a_{k}\right)^{-\frac{1}{d-2}} a\right)^{\frac{2(d-2)}{d-1}}\left(\left(a_{j} a_{k}\right)^{\frac{1}{d-2}} r\right)^{(d-2)-\frac{2(d-2)}{d-1}} .
\end{aligned}
$$

Observe that the total contribution of $a_{j}$ and $a_{k}$ to the right hand side is

$$
\left(a_{j} a_{k}\right)^{\left(-\frac{1}{d-2}\right)\left(\frac{2(d-2)}{d-1}+(d-2)-\frac{2(d-2)}{d-1}\right)}=a_{j}^{-1} a_{k}^{-1} .
$$

Therefore, bounding $a_{j}^{-1} a_{k}^{-1}$ by $a^{2}$ yields the bound

$$
\left|\#\left\{n \in \mathbb{Z}^{d} \cap A_{j, k}\left(r \Omega_{j, k}\right)\right\}-\right| \Omega_{j, k}\left|a_{j}^{-1} a_{k}^{-1} r^{d-2}\right| \lesssim_{\Omega} a^{2}\left(a^{\frac{2(d-2)}{d-1}} r^{(d-2)-\frac{2(d-2)}{d-1}}\right) .
$$

A direct computation yields:

$$
\left(a^{\frac{2 d}{d+1}} r^{d-\frac{2 d}{d+1}}\right)\left(a^{\frac{2(d-2)}{d-1}} r^{(d-2)-\frac{2(d-2)}{d-1}}\right)^{-1}=a^{\frac{4}{d^{2}-1}} r^{\frac{2 d^{2}-6}{d^{2}-1}}
$$

Therefore, since we have assumed $a \leq C r$, it follows that

Similarly, it follows that

$$
a^{2}\left(a^{\frac{2(d-2)}{d-1}} r^{(d-2)-\frac{2(d-2)}{d-1}}\right) \lesssim \Omega a^{\frac{2 d}{d+1}} r^{d-\frac{2 d}{d+1}}
$$

Therefore, we conclude that

$$
\left|\Omega_{j, k}\right| a_{j}^{-1} a_{k}^{-1} r^{d-2} \lesssim \Omega a^{\frac{2 d}{d+1}} r^{d-\frac{2 d}{d+1}}
$$

$$
\#\left\{n \in \mathbb{Z}^{d} \cap A_{j, k}\left(r \Omega_{j, k}\right)\right\} \lesssim \Omega^{\frac{2 d}{d+1}} r^{d-\frac{2 d}{d+1}} .
$$

Summing over $1 \leq j<k \leq d$ gives

$$
\sum_{1 \leq j<k \leq d} \#\left\{n \in \mathbb{Z}^{d} \cap A_{j, k}\left(r \Omega_{j, k}\right)\right\} \lesssim a^{\frac{2 d}{d+1}} r^{d-\frac{2 d}{d+1}}
$$


Recall that we previously established the inequality

$$
\left|\sum_{j=1}^{d} \#\left\{n \in \mathbb{Z}^{d} \cap A_{j}\left(r \Omega_{j}\right)\right\}-\left(\operatorname{tr} A^{-1}\right) r^{d-1}\right| \lesssim_{\Omega} a^{\frac{2 d}{d+1}} r^{d-\frac{2 d}{d+1}} .
$$

These last two inequalities can be used to deduce the result in combination with the observation that:

$$
\sum_{j=1}^{d} \#\left\{n \in \mathbb{Z}^{d} \cap A_{j}\left(r \Omega_{j}\right)\right\}-\sum_{1 \leq j<k \leq d} \#\left\{n \in \mathbb{Z}^{d} \cap A_{j, k}\left(r \Omega_{j, k}\right)\right\} \leq \#\left\{n \in \mathbb{Z}^{d} \cap A\left(r \bigcup_{j=1}^{d} \Omega_{j}\right)\right\},
$$

and

$$
\#\left\{n \in \mathbb{Z}^{d} \cap A\left(r \bigcup_{j=1}^{d} \Omega_{j}\right)\right\} \leq \sum_{j=1}^{d} \#\left\{n \in \mathbb{Z}^{d} \cap A_{j}\left(r \Omega_{j}\right)\right\} .
$$

Step 3.3. Assume that $\Omega$ is balanced in the sense of Definition 2.1 and is symmetric with respect to each coordinate hyperplane. Then

$$
\#\left\{n \in \mathbb{Z}_{>0}^{d} \cap A(r \Omega)\right\}=\frac{1}{2^{d}}|\Omega| r^{d}-\frac{1}{2^{d}}\left(\operatorname{tr} A^{-1}\right) r^{d-1}+\mathcal{O}\left(a^{\frac{2 d}{d+1}} r^{d-\frac{2 d}{d+1}}\right) .
$$

Proof. We partition the proof into two cases:

$$
1 \leq a \leq C r, \quad \text { and } \quad C r<a<\infty .
$$

Case 1. If $1 \leq a \leq C r$, then the results from Steps 3.1 and 3.2 hold. By the assumed symmetry of the domain, the number of positive lattice points contained in $\Omega$ is equal to the number of lattice points in $\Omega$ minus those in $\bigcup_{j=1}^{d} \Omega_{j}$ divided by $2^{d}$ :

$$
\#\left\{n \in \mathbb{Z}_{>0}^{d} \cap A(r \Omega)\right\}=\frac{1}{2^{d}}\left(\#\left\{n \in \mathbb{Z}^{d} \cap A(r \Omega)\right\}-\#\left\{n \in \mathbb{Z}^{d} \cap A\left(r \bigcup_{j=1}^{d} \Omega_{j}\right)\right\}\right) .
$$

Combing the results of Steps 3.1 and 3.2 yields the result.

Case 2. If $C r<a<\infty$, then we argue as follows. Recall that $C>0$ is a constant such that $\Omega \subset[-C, C]^{d}$. Therefore, if $a>C r$, then $a_{1}<1 /(C r)$ and hence

$$
A(r \Omega) \subseteq(-1,1) \times \mathbb{R}^{d-1} .
$$

In particular, it follows that

$$
\#\left\{n \in \mathbb{Z}_{>0}^{d} \cap A(r \Omega)\right\}=0 .
$$

Therefore, the statement to prove reduces to

$$
0=\frac{1}{2^{d}}|\Omega| r^{d}-\frac{1}{2^{d}}\left(\operatorname{tr} A^{-1}\right) r^{d-1}+\mathcal{O}\left(a^{\frac{2 d}{d+1}} r^{d-\frac{2 d}{d+1}}\right),
$$

which trivially holds because the error term dominates the right hand side since $\mathrm{Cr}<a<\infty$.

Step 3.4. Suppose that $\Omega$ is balanced in the sense of Definition 2.1 and is symmetric with respect to each coordinate hyperplane. Let

$$
A(r)=\underset{A}{\operatorname{argmax}} \#\left\{n \in \mathbb{Z}_{>0}^{d} \cap A(r \Omega)\right\},
$$

where the argmax ranges over all positive diagonal matrices $A$ of determinant 1 . Write $A(r)=$ $\operatorname{diag}\left(a_{1}(r), a_{2}(r), \ldots, a_{d}(r)\right)$. Without loss of generality suppose $a_{1}(r) \leq a_{2}(r) \leq \cdots \leq a_{d}(r)$ and let $a(r)=1 / a_{1}(r)=\left\|A^{-1}(r)\right\|_{\infty}$. Then

$$
\lim _{r \rightarrow \infty} a(r)=1
$$


Remark 3.1. Two technical remarks are in order about the definition of $A(r)$. First, we argue why the argmax exists. As noted in Step 3.3, if $\mathrm{Cr}<a<\infty$, then $A(r \Omega)$ will not contain any positive lattice points; therefore, the admissible $A$ for the argmax can be restricted to those satisfying

$$
1 \leq\left\|A^{-1}\right\|_{\infty} \leq C r \Longrightarrow A(r \Omega) \subset\left[-(C r)^{d},(C r)^{2}\right]^{d},
$$

where $C>0$ is a constant such that $\Omega \subset[-C, C]^{d}$. Since the set $\left[-(C r)^{2},(C r)^{2}\right]^{d}$ contains finitely many positive lattice points, we conclude that the argmax exists. Second, when the argmax is not unique, $A(r)$ should be interpreted as being equal to an arbitrary element from the maximal set; all convergence results are independent of this choice.

Proof of Step 3.4. By the argument in Case 2 of Step 3.3 , we may assume $1 \leq a(r) \leq C r$. First, we will suppose that there exists a constant $c>0$ such that

$$
\frac{1}{c} r \leq a(r) \leq C r
$$

for arbitrarily large values $r$. That is to say, we suppose that there exists a sequence $r_{n}$ tending towards infinity such that $r_{n} / c \leq a\left(r_{n}\right) \leq C r_{n}$. We show that this assumption leads to a contradiction, which implies that

$$
a(r) / r \rightarrow 0, \quad \text { as } r \rightarrow \infty .
$$

While producing this contradiction, we write $A=A(r)$ and $a=a(r)$ to simplify notation. Let $\chi$ denote the indicator function for $\Omega$. Then

$$
\#\left\{n \in \mathbb{Z}_{>0}^{d} \cap A(r \Omega)\right\}=\sum_{n \in \mathbb{Z}_{>0}^{d}} \chi\left(A^{-1} n / r\right) .
$$

Let $\left(n_{1}, n^{\prime}\right) \in \mathbb{Z}_{>0} \times \mathbb{Z}_{>0}^{d-1}$, and let $A_{1}$ denote the $(d-1) \times(d-1)$ matrix formed by removing the 1 -st row and 1-st column from $A$. With this notation

$$
\#\left\{n \in \mathbb{Z}_{>0}^{d} \cap A(r \Omega)\right\}=\sum_{n_{1} \in \mathbb{Z}_{>0}} \sum_{n^{\prime} \in \mathbb{Z}_{>0}^{d-1}} \chi\left(\frac{n_{1}}{a_{1} r}, \frac{A_{1}^{-1} n^{\prime}}{r}\right) .
$$

Since by assumption $r / c \leq a \leq C r$, and $a=1 / a_{1}$, we have

$$
\frac{1}{C} \leq r a_{1} \leq c .
$$

For simplicity, assume $r a_{1}=c$. In the course of the proof, we will only use the fact that $r a_{1}$ is bounded above by a fixed constant. We remark that the method of estimating the sum in this part of the proof is similar to the arguments in [5, 18. With the notation $r a_{1}=c$

$$
\#\left\{n \in \mathbb{Z}_{>0}^{d} \cap A(r \Omega)\right\}=\sum_{n_{1} \in \mathbb{Z}_{>0}} \sum_{n^{\prime} \in \mathbb{Z}_{>0}^{d-1}} \chi\left(\frac{n}{c}, \frac{A_{1}^{-1} n^{\prime}}{r}\right) .
$$

We assert that:

$$
\sum_{n^{\prime} \in \mathbb{Z}_{>0}^{d-1}} \chi\left(\frac{n}{c}, \frac{A_{1}^{-1} n^{\prime}}{r}\right) \leq \int_{\mathbb{R}_{\geq 0}^{d-1}} \chi\left(\frac{n_{1}}{c}, \frac{A_{1}^{-1} x^{\prime}}{r}\right) d x^{\prime} .
$$

Indeed, each lattice point $\left(n_{1}, n^{\prime}\right)$ can be identified with the $(d-1)$-dimensional cube with sides parallel to the coordinates axes and vertices $\left(n_{1}, n^{\prime}\right)$ and $\left(n_{1}, n^{\prime}-\overrightarrow{1}\right)$, where $\overrightarrow{1}$ denotes a $(d-1)$ dimensional vector of ones. Since the set $A(r \Omega)$ is convex, each of these cubes is contained in $A(r \Omega)$ and their first coordinate is equal to $n_{1}$. Moreover, this collection of cubes is disjoint since each pair of vertices is unique. Therefore, the sum is a lower approximation of the integral. Thus we have

$$
\#\left\{n \in \mathbb{Z}_{>0}^{d} \cap A(r \Omega)\right\} \leq \sum_{n_{1} \in \mathbb{Z}_{>0}} \int_{\mathbb{R}_{\geq 0}^{d-1}} \chi\left(\frac{n_{1}}{c}, \frac{A_{1}^{-1} x^{\prime}}{r}\right) d x^{\prime} .
$$


Define the function $f_{A_{1}, r}: \mathbb{R} \rightarrow \mathbb{R}$ by

$$
f_{A_{1}, r}\left(x_{1}\right)=\int_{\mathbb{R}_{\geq 0}^{d-1}} \chi\left(\frac{x_{1}}{c}, \frac{A_{1}^{-1} x^{\prime}}{r}\right) d x^{\prime} .
$$

The function $f_{A_{1}, r}\left(x_{1}\right)$ is supported on some interval $[0, b]$ such that $0<b<c C$, where $C>0$ is a constant such that $\Omega \subset[-C, C]^{d}$. Define

$$
\Omega_{x_{1} / c}:=\Omega \cap\left\{\frac{x_{1}}{c}\right\} \times \mathbb{R}^{d-1} \quad \text { such that } f_{A_{1}, r}\left(x_{1}\right)=\frac{1}{2^{d-1}}\left|A_{1}\left(r \Omega_{x_{1} / c}\right)\right|,
$$

where $|\cdot|$ denotes the $(d-1)$-dimensional Lebesgue measure, and where the factor $1 / 2^{d-1}$ arises since the integral is taken over $\mathbb{R}_{\geq 0}^{d-1}$. Since $\operatorname{det}\left(A_{1}\right) \cdot a_{1}=1$ and $a_{1}=c / r$ it follows that

$$
f_{A_{1}, r}\left(x_{1}\right)=\frac{1}{2^{d-1}} \frac{r}{c}\left|r \Omega_{x_{1} / c}\right|=\frac{1}{2^{d-1}} r^{d} \frac{1}{c}\left|\Omega_{x_{1} / c}\right| .
$$

Since $\Omega$ is symmetric about the coordinate axes and is strictly convex, the maximum value of $f_{A_{1}, r}\left(x_{1}\right)$ occurs at $x_{1}=0, f_{A_{1}, r}\left(x_{1}\right)$ is strictly decreasing on $[0, b]$, and $f_{A_{1}, r}(b)=0$. Moreover, since we assume the boundary of $\Omega$ is $C^{d+2}$ the function $f_{A_{1}, r}$ is certainly $C^{1}$, which is sufficient for our purposes. Integrating $f_{A_{1}, r}$ on $[0, b]$ yields

$$
\int_{0}^{b} f_{A_{1}, r}\left(x_{1}\right) d x_{1}=\frac{1}{2^{d-1}} r^{d} \int_{0}^{b} \frac{1}{c}\left|\Omega_{x_{1} / c}\right| d x_{1}=\frac{1}{2^{d}}|\Omega| r^{d} .
$$

However, our previous arguments show that

$$
\#\left\{n \in \mathbb{Z}_{>0}^{d} \cap A(r \Omega)\right\} \leq \sum_{n_{1} \in \mathbb{Z}_{>0}} f_{A_{1}, r}\left(x_{1}\right)=\frac{1}{2^{d-1}} r^{d} \sum_{n_{1}=1}^{\lfloor b\rfloor} \frac{1}{c}\left|\Omega_{x_{1} / c}\right| .
$$

We assert that there exists $\varepsilon>0$ such that

$$
\sum_{n_{1}=1}^{\lfloor b\rfloor} \frac{1}{c}\left|\Omega_{n_{1} / c}\right| \leq(1-\varepsilon) \int_{0}^{b} \frac{1}{c}\left|\Omega_{x_{1} / c}\right| d x_{1} .
$$

Indeed, the sum on the left hand side is a lower Riemann sum for the integral of the strictly decreasing $C^{1}$ function $(1 / c)\left|\Omega_{x_{1} / c}\right|$. Moreover, the constant $\varepsilon>0$ can be chosen to hold uniformly for any lower Riemann sum of the integral which discretizes the integral into at most $\lfloor c C\rfloor$ pieces.

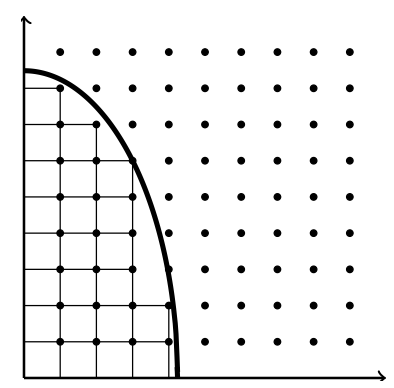

FiguRE 7. An approximation of the area enclosed by an ellipse by squares $\left[i_{1}-1, i_{1}\right] \times\left[i_{2}-1, i_{2}\right]$.

Therefore, we conclude that

$$
\#\left\{n \in \mathbb{Z}_{>0}^{d} \cap A(r \Omega)\right\} \leq \sum_{n_{1}=1}^{\lfloor b\rfloor} f_{A_{1}, r}\left(n_{1}\right) \leq(1-\varepsilon) \int_{0}^{b} f_{A_{1}, r}\left(x_{1}\right) d x_{1}=(1-\varepsilon) \frac{1}{2^{d}}|\Omega| r^{d} .
$$

However, applying Step 3.3 with $A=$ Id yields

$$
\#\left\{n \in \mathbb{Z}_{>0}^{d} \cap \operatorname{Id}(r \Omega)\right\}=\frac{1}{2^{d}}|\Omega| r^{d}-\frac{1}{2^{d}} d r^{d-1}+\mathcal{O}\left(r^{d-\frac{2 d}{d+1}}\right) .
$$


When $r$ is sufficiently large

$$
-\varepsilon r^{d}<-\frac{1}{2^{d}} d r^{d-1}+\mathcal{O}\left(r^{d-\frac{2 d}{d+1}}\right)
$$

which contradicts the optimality of such $a \geq r / c$. Therefore, we conclude that

$$
a(r)=\psi(r) r, \quad \text { where } \quad \psi(r) \rightarrow 0, \quad \text { as } \quad r \rightarrow \infty .
$$

By the Step 3.3 of the proof:

$$
\#\left\{n \in \mathbb{Z}_{>0}^{d} \cap A(r)(r \Omega)\right\}=\frac{1}{2^{d}}|\Omega| r^{d}-\frac{1}{2^{d}}\left(\operatorname{tr} A(r)^{-1}\right) r^{d-1}+\mathcal{O}\left(a(r)^{\frac{2 d}{d+1}} r^{d-\frac{2 d}{d+1}}\right) .
$$

Substituting $a(r)=\psi(r) r$ yields

$$
\#\left\{n \in \mathbb{Z}_{>0}^{d} \cap A(r)(r \Omega)\right\} \leq \frac{1}{2^{d}}|\Omega| r^{d}-\left(\frac{1}{2^{d}}-C_{1} \psi(r)^{\frac{d-1}{d+1}}\right) \psi(r) r^{d},
$$

for some constant $C_{1}$. Since $\psi(r) \rightarrow 0$, we may choose $r$ large enough such that

$$
\frac{1}{2^{d}}-C_{1} \psi(r)^{\frac{d-1}{d+1}} \geq \frac{1}{2^{d+1}}
$$

Thus for large enough $r$,

$$
\#\left\{n \in \mathbb{Z}_{>0}^{d} \cap A(r)(r \Omega)\right\} \leq \frac{1}{2^{d}}|\Omega| r^{d}-\frac{1}{2^{d+1}} \psi(r) r^{d} .
$$

However, if such a situation is optimal, it must be competitive with the situation $A=\mathrm{Id}$, where

$$
\#\left\{n \in \mathbb{Z}_{>0}^{d} \cap \operatorname{Id}(r \Omega)\right\}=\frac{1}{2^{d}}|\Omega| r^{d}-\frac{d}{2^{d}} r^{d-1}+\mathcal{O}\left(r^{d-\frac{2 d}{d+1}}\right) .
$$

Therefore, we conclude that

which implies

$$
\psi(r) r^{d}=\mathcal{O}\left(r^{d-1}\right)
$$

$$
\psi(r)=\mathcal{O}\left(r^{-1}\right)
$$

Therefore, the set of optimal $a$ is uniformly bounded. Convergence then immediately follows from the result from Step 3.3 , i.e., from the equation:

$$
\#\left\{n \in \mathbb{Z}_{>0}^{d} \cap A(r \Omega)\right\}=\frac{1}{2^{d}}|\Omega| r^{d}-\frac{1}{2^{d}}\left(\operatorname{tr} A^{-1}\right) r^{d-1}+\mathcal{O}\left(a^{\frac{2 d}{d+1}} r^{d-\frac{2 d}{d+1}}\right) .
$$

Indeed, since $a$ is uniformly bounded the second term determines the effect of $A$ when $r$ is large. Therefore, in order to maximize $\#\left\{n \in \mathbb{Z}_{>0}^{d} \cap A(r \Omega)\right\}$ the coefficient of $r^{d-1}$ must be minimized. More precisely, $A(r)$ can be characterized in terms of the following minimization problem

$$
A(r)=\underset{A}{\operatorname{argmin}}\left(\operatorname{tr} A^{-1}\right)+\mathcal{O}\left(r^{-\frac{d-1}{d+1}}\right)
$$

where the argmin is taken over positive diagonal matrices $A$ of determinant 1 such that $1 \leq$ $\left\|A^{-1}\right\|_{\infty} \leq c_{0}$, where $c_{0} \geq 1$ is a fixed constant. By the arithmetic mean geometric mean inequality, any positive diagonal matrix $A$ of determinant 1 satisfies

$$
\operatorname{tr} A^{-1} \geq d\left(\operatorname{det} A^{-1}\right)^{1 / d}=d
$$

with equality if and only if $A=\mathrm{Id}$. Therefore, we conclude that

$$
\operatorname{tr} A(r)^{-1} \rightarrow d, \quad \text { as } r \rightarrow \infty .
$$

Since $\operatorname{tr} A^{-1}$ is a continuous function, and equality holds in the arithmetic mean geometric mean inequality if and only if $A=\mathrm{Id}$ we conclude

$$
\|A(r)-\mathrm{Id}\|_{\infty} \rightarrow 0, \quad \text { as } r \rightarrow \infty
$$

as was to be shown.

In the fifth step, we establish a rate of convergence. 
Step 3.5. Suppose that $\Omega$ is balanced in the sense of Definition 2.1 and is symmetric with respect to each coordinate hyperplane. Let

$$
A(r)=\underset{A}{\operatorname{argmax}} \#\left\{n \in \mathbb{Z}_{>0}^{d} \cap A(r \Omega)\right\},
$$

where the argmax ranges over all positive diagonal matrices $A$ of determinant 1 . Then

$$
\|A(r)-\mathrm{Id}\|_{\infty}=\mathcal{O}\left(r^{-\frac{d-1}{2(d+1)}}\right) .
$$

Proof. Applying the result of Step 3.3 for $A=$ Id gives

$$
\#\left\{n \in \mathbb{Z}_{>0}^{d} \cap \operatorname{Id}(r \Omega)\right\}=\frac{1}{2^{d}}|\Omega| r^{d}-\frac{1}{2^{d}} d r^{d-1}+\mathcal{O}\left(r^{d-\frac{2 d}{d+1}}\right) .
$$

By the arithmetic mean geometric mean inequality

$$
\operatorname{tr} A^{-1} \geq d\left(\operatorname{det} A^{-1}\right)^{1 / d}=d .
$$

Therefore, since $A(r)$ maximizes $\#\left\{n \in \mathbb{Z}_{>0}^{d} \cap A(r \Omega)\right\}$ over all $A$, we must have

$$
\frac{1}{2^{d}} \operatorname{tr} A^{-1}(r) r^{d-1}-\frac{1}{2^{d}} d r^{d-1} \lesssim_{\Omega} r^{d-\frac{2 d}{d+1}},
$$

which simplifies to

$$
\operatorname{tr} A^{-1}(r)-d \lesssim \Omega r^{-\frac{d-1}{d+1}}
$$

To complete the proof it suffices to show that:

$$
\frac{1}{8}\|A-\operatorname{Id}\|_{\infty}^{2} \leq \operatorname{tr} A^{-1}-d .
$$

Indeed then,

$$
\frac{1}{8}\|A-\mathrm{Id}\|_{\infty}^{2} \leq \operatorname{tr} A^{-1}-d \lesssim_{\Omega} r^{-\frac{d-1}{d+1}} \quad \Longrightarrow \quad\|A-\mathrm{Id}\|_{\infty} \lesssim_{\Omega} r^{-\frac{d-1}{2(d+1)}} .
$$

Without loss of generality, suppose that

$$
A(r)=\operatorname{diag}\left(a_{1}, a_{2}, \ldots, a_{d}\right) \quad \text { where } \quad a_{1} \leq a_{2} \leq \ldots \leq a_{d} .
$$

There are two cases to consider

Case 1. $\|A(r)-\operatorname{Id}\|_{\infty}=\left|a_{d}-1\right|$. In this case, suppose that $a_{d}=1+\varepsilon$ where $\varepsilon>0$. Then

$$
\|A(r)-\mathrm{Id}\|_{\infty}=\varepsilon .
$$

Since the determinant of $A$ is equal 1 , it follows that the product $a_{1} \cdot a_{2} \cdots \cdots a_{d-1}=\frac{1}{1+\varepsilon}$. Therefore, by the arithmetic mean geometric mean inequality,

$$
(d-1)(1+\varepsilon)^{\frac{1}{d-1}}+\left(\frac{1}{1+\varepsilon}\right) \leq \frac{1}{a_{1}}+\frac{1}{a_{2}}+\cdots \frac{1}{a_{d-1}}+\left(\frac{1}{1+\varepsilon}\right)=\operatorname{tr} A^{-1} .
$$

Expanding the left hand side in a Taylor series yields

$$
d+\frac{d \varepsilon^{2}}{2(d-1)}+\mathcal{O}\left(\varepsilon^{3}\right)=\operatorname{tr} A^{-1} .
$$

And therefore, when $\varepsilon$ is sufficiently small,

$$
\frac{1}{2}\|A(r)-\operatorname{Id}\|_{\infty}^{2}=\frac{1}{2} \varepsilon^{2} \leq \operatorname{tr} A^{-1}-d .
$$


Case 2. $\|A(r)-\operatorname{Id}\|_{\infty}=\left|a_{1}-1\right|$. Suppose that $a_{1}=\frac{1}{1+\varepsilon}$. When $\varepsilon$ is sufficiently small

$$
\|A(r)-\operatorname{Id}\|_{\infty}=\left|a_{1}-1\right| \leq 2 \varepsilon .
$$

Furthermore, since the determinant of $A$ is equal to 1

$$
a_{2} \cdot a_{3} \cdots a_{d}=1+\varepsilon .
$$

Therefore, by the arithmetic mean geometric mean inequality

$$
(1+\varepsilon)+(d-1)\left(\frac{1}{1+\varepsilon}\right)^{\frac{1}{d-1}} \leq(1+\varepsilon)+\frac{1}{a_{2}}+\frac{1}{a_{3}}+\cdots \frac{1}{a_{d}}=\operatorname{tr} A^{-1} .
$$

Expanding the left hand side in a Taylor series yields

$$
d+\frac{d \varepsilon^{2}}{2(d-1)}+\mathcal{O}\left(\varepsilon^{3}\right) \leq \operatorname{tr} A^{-1} .
$$

Therefore, when $\varepsilon$ is sufficiently small,

Moreover, in either case

$$
\frac{1}{8} \| A-\left.\mathrm{Id}\right|^{2} \leq \frac{1}{2} \varepsilon^{2} \leq \operatorname{tr} A^{-1}-d
$$

and the proof is complete.

$$
\frac{1}{8}\|A-\operatorname{Id}\|^{2} \leq \operatorname{tr} A^{-1}-d
$$

Acknowledgment. We are grateful to Stefan Steinerberger for many useful discussions, and Andrei Deneanu for insightful comments. Additionally, we would like to thank Richard Laugesen for valuable feedback leading to corrections in the proof and a much improved exposition, and the referees for their helpful comments.

\section{REFERENCES}

[1] P. R. S. Antunes and P. Freitas. Optimal spectral rectangles and lattice ellipses. Proc. Roy. Soc. London Ser. A, 469(2150), 2012.

[2] P. R. S. Antunes and P. Freitas. Optimisation of eigenvalues of the Dirichlet Laplacian with a surface area restriction. Appl. Math. Optim., 73(2):313-328, 2016.

[3] S. Ariturk and R. S. Laugesen. Optimal stretching for lattice points under convex curves. Port. Math., 74(2):91$114,2017$.

[4] D. Bucur and P. Freitas. Asymptotic behaviour of optimal spectral planar domains with fixed perimeter. $J$. Math. Phys., 54(5):053504, 2013.

[5] K. Gittins and S. Larson. Asymptotic behaviour of cuboids optimising Laplacian eigenvalues. S. Integr. Equ. Oper. Theory, 89(4):607-629, 2017.

[6] F. Götze. Lattice point problems and values of quadratic forms. Invent. Math., 157:195-225, 2004.

[7] M. N. Huxley. Exponential sums and lattice points III. Proc. London Math. Soc., 87:591, 2003.

[8] V. Ivrii. 100 years of Weyl's law. Bull. Math. Sci., 6(3):379-452, 2016.

[9] V. Ivrii. On the second term of the spectral asymptotics for the Laplace-Beltrami operator on manifolds with boundary. Funktsional. Anal. i Prilozhen., 14(2):25-24, 1980.

[10] R. Laugesen and S. Liu. Optimal stretching for lattice points and eigenvalues. ArXiv e-prints (to appear in Ark. Mat.), September 2016.

[11] N. F. Marshall and S. Steinerberger. Triangles capturing many lattice points. ArXiv e-prints, June 2017.

[12] W. Sierpiński. O pewnem zagadneniu w rachunku funkcyj asymptotznych. Prace Mat. Fiz, 17:77-118, 1906.

[13] E. M. Stein. Harmonic Analysis: Real-Variable Methods, Orthogonality, and Oscillatory Integrals. Princeton University Press, Princeton, New Jersey, 1993.

[14] E. M. Stein and Rami Shakarchi. Princeton Lectures in Analysis: Functional Analysis, Princeton University Press, Princeton, New Jersey, 2011.

[15] G. Travaglini. Average Decay of the Fourier Transform. In L. Brandolini, L. Colzani, A. Iosevich, G. Travaglini, editors. Fourier Analysis and Convexity, pages 245-268, Birkhäuser, 2004.

[16] M. van den Berg. On the minimization of Dirichlet eigenvalues. Bull. Lond. Math. Soc., 47(1):143-155, 2015.

[17] M. van den Berg, D. Bucur, and K. Gittins. Maximising Neumann eigenvalues on rectangles. Bull. Lond. Math. Soc., 48(5):877-894, 2016.

[18] M. van den Berg and K. Gittins. Minimising Dirichlet eigenvalues on cuboids of unit measure. Mathematika, 63:469-482, 2017.

[19] J. G. van der Corput. Neue zahlentheoretische Abschätzungen. Math. Ann., 89:215-254, 1923. 
[20] G. Voronoi. Sur un problème du calcul des fonctions asymptotiques. J. Reine Angew. Math., 126:241-282, 1903.

[21] H. Weyl. Über die Randwertaufgabe der Strahlungstheorie und asymptotische Spektralgesetze. J. Reine Angew. Math., 143(3):177-202, 1913.

Department of Mathematics, Yale University, New Haven, CT 06511, USA

E-mail address: nicholas.marshall@yale.edu 